\title{
A longitudinal analysis of the impact of the COVID-19 pandemic on the mental health of middle-aged and older adults from the Canadian Longitudinal Study on Aging
}

\author{
Parminder Raina ${ }^{1,2,3,4} \bowtie$, Christina Wolfson ${ }^{5,6,7}$, Lauren Griffith ${ }^{1,2,3}$, Susan Kirkland ${ }^{8}$, \\ Jacqueline McMillan ${ }^{\circledR}{ }^{9}$, Nicole Basta5 ${ }^{5}$, Divya Joshi ${ }^{1,2,3}$, Urun Erbas Oz ${ }^{1}$, Nazmul Sohel', \\ Geva Maimon ${ }^{10}$, Mary Thompson ${ }^{11}$ and CLSA team*
}

\begin{abstract}
The coronavirus disease 2019 (COVID-19) pandemic presents an unprecedented challenge to public health, with over 233 million confirmed cases and over $\mathbf{4 . 6}$ million deaths globally as of September 2021'. Although many studies have reported worse mental health outcomes during the early weeks of the pandemic, some sources suggest a gradual decrease in anxiety and depressive symptoms during the lockdown ${ }^{2}$. It remains to be explained whether mental health continued to deteriorate during the initial lockdown or whether there were signs of stabilization or improvement in the mental health of communitydwelling middle-aged and older adults. Our results showed that adults had twice the odds of depressive symptoms during the pandemic compared with the prepandemic period, with subgroups characterized by lower socioeconomic status and poor health-related factors experiencing a greater impact. Over $43 \%$ of adults showed a pattern of moderate or clinically high levels of depressive symptoms at baseline that increased over time. Loneliness and COVID-19 stressors were predictors of worsening depressive symptom trajectories. The disparities and patterns in the depressive symptom trajectories suggest that the negative mental health impacts of the pandemic persist and may worsen over time. Interventions that address the pandemic stressors and alleviate their impact on the mental health of adults are needed.

The rapid spread of the severe acute respiratory syndrome coronavirus 2 (SARS-CoV-2) virus across the globe resulted in many national and local governments implementing drastic health measures, including isolation, quarantine, lockdowns, physical distancing, closure of nonessential services and travel restrictions, to contain the spread of the virus ${ }^{3}$. The first complete lockdown began in most Canadian provinces in mid-March 2020 and continued until June 2020, with large numbers of people across the country urged to stay at home for substantial periods of time. The direct
\end{abstract}

risks of the virus, uncertainty about disease status and the socioeconomic consequences of the enacted public health measures can have a substantial, long-lasting impact on individuals' mental health and well-being, especially for older adults who are disproportionately affected by COVID-19, and the pandemic mitigation measures ${ }^{4}$.

Studies comparing mental health outcomes before the pandemic with results obtained during the early weeks of the pandemic have shown an increase in clinically significant levels of psychological distress ${ }^{5,6}$. Evidence from previous outbreaks, including the severe acute respiratory syndrome (SARS) and Ebola outbreaks, and the current COVID-19 pandemic indicates that many individuals have experienced a wide range of adversities, including challenges with meeting basic needs, increased caregiving responsibilities, difficulties with accessing non-COVID-19-related healthcare, employment and financial loss, and disruption of social networks, which may increase the risk of mental illness during and after the outbreaks ${ }^{7-9}$. Further, the impact of the COVID-19 pandemic on the older population has occurred against the backdrop of existing physical and mental health morbidities, social isolation, loneliness and reliance of aging individuals on both family and formal caregivers-factors that are themselves associated with increased risk, severity and progression of mental illness ${ }^{10}$. These findings highlight the importance of identifying subgroups of individuals who are most at risk of poor mental health and examining how their mental health is changing as the pandemic continues.

Most studies examining the mental health impact of the COVID19 pandemic have been conducted in younger samples, have included specific target populations and have been cross-sectional in design. Some longitudinal studies have focused on average change in depressive symptoms for the entire sample or lacked depression measures before the pandemic, which makes it challenging to examine changes in mental health and can obscure different patterns of change in mental health over time. Although many studies

'Department of Health Research Methods, Evidence, and Impact, McMaster University, Hamilton, Ontario, Canada. ${ }^{2}$ Labarge Centre for Mobility in Aging, McMaster University, Hamilton, Ontario, Canada. ${ }^{3}$ McMaster Institute for Research on Aging, McMaster University, Hamilton, Ontario, Canada. ${ }^{4}$ Department of Psychiatry and Behavioral Neurosciences, McMaster University, Hamilton, Ontario, Canada. ${ }^{5}$ Department of Epidemiology, Biostatistics and Occupational Health, McGill University, Montreal, Quebec, Canada. ${ }^{6}$ Department of Medicine, McGill University, Montreal, Quebec, Canada. ${ }^{7}$ Research Institute of the McGill University Health Centre, McGill University, Montreal, Quebec, Canada. ${ }^{8}$ Department of Community Health and Epidemiology, Dalhousie University, Halifax, Nova Scotia, Canada. ${ }^{9}$ Departments of Medicine, Faculty of Medicine, University of Calgary, Calgary, Alberta, Canada. ${ }^{10} \mathrm{CLSA}$ Data Curation Centre, Research Institute of the McGill University Health Centre, Montreal, Quebec, Canada. ${ }^{11}$ Department of Statistics and Actuarial Science, University of Waterloo, Waterloo, Ontario, Canada. ${ }^{\star} A$ list of authors and their affiliations appears at the end of the paper.

凶e-mail: praina@mcmaster.ca 
Table 1 | Depressive symptom prevalence by sociodemographic characteristics and health behaviors before and during the pandemic $(n=22,875)^{a}$

\begin{tabular}{|c|c|c|c|c|}
\hline Characteristic or behavior & CLSA baseline & CLSA first follow-up & CLSA COVID-19 baseline & CLSA COVID-19 exit \\
\hline \multicolumn{5}{|l|}{ Age group } \\
\hline$<55$ years & 14.47 & 13.84 & 26.72 & 29.39 \\
\hline $55-64$ years & 13.56 & 12.44 & 22.95 & 23.21 \\
\hline $65-74$ years & 12.01 & 11.64 & 18.52 & 20.58 \\
\hline$\geq 75$ years & 12.70 & 15.00 & 18.66 & 20.71 \\
\hline \multicolumn{5}{|l|}{ Sex } \\
\hline Female & 15.98 & 15.71 & 25.08 & 26.02 \\
\hline Male & 10.15 & 9.60 & 14.82 & 16.82 \\
\hline \multicolumn{5}{|l|}{ Ethnicity } \\
\hline White & 12.92 & 12.61 & 20.12 & 21.61 \\
\hline Nonwhite & 18.35 & 16.06 & 23.01 & 23.94 \\
\hline \multicolumn{5}{|l|}{ Annual household income ${ }^{b}$} \\
\hline$<\$ 20,000$ & 31.99 & 31.94 & 33.96 & 31.57 \\
\hline$\geq \$ 20,000$ to $<\$ 50,000$ & 17.86 & 18.72 & 23.89 & 25.73 \\
\hline$\geq \$ 50,000$ to $<\$ 100,000$ & 12.43 & 11.87 & 19.09 & 21.05 \\
\hline$\geq \$ 100,000$ to $<\$ 150,000$ & 9.87 & 9.24 & 18.16 & 19.29 \\
\hline$\geq \$ 150,000$ & 7.84 & 6.77 & 17.37 & 17.79 \\
\hline \multicolumn{5}{|l|}{ Social participation ${ }^{b}$} \\
\hline Low social participation & 21.42 & 21.23 & 25.49 & 28.04 \\
\hline High social participation & 11.45 & 10.93 & 19.10 & 20.29 \\
\hline \multicolumn{5}{|c|}{ Number of people living in the same household ${ }^{c}$} \\
\hline Living alone & 19.69 & 19.70 & 26.97 & 27.71 \\
\hline Not living alone & 11.58 & 10.80 & 18.03 & 19.61 \\
\hline \multicolumn{5}{|l|}{ Dwelling type ${ }^{c}$} \\
\hline House & 12.05 & 11.43 & 18.98 & 20.18 \\
\hline Apartment or condominium & 19.26 & 18.64 & 24.28 & 26.98 \\
\hline Other & 24.48 & 21.49 & 28.32 & 28.43 \\
\hline \multicolumn{5}{|l|}{ Living area ${ }^{c}$} \\
\hline Rural & 12.52 & 12.44 & 18.98 & 18.81 \\
\hline Urban & 13.40 & 12.91 & 20.59 & 22.39 \\
\hline \multicolumn{5}{|l|}{ Number of chronic conditions ${ }^{b}$} \\
\hline None & 8.57 & 7.41 & 14.38 & 15.59 \\
\hline One & 10.87 & 7.56 & 15.69 & 16.79 \\
\hline Two & 13.71 & 10.41 & 18.16 & 19.47 \\
\hline Three or more & 18.68 & 17.96 & 24.96 & 26.70 \\
\hline \multicolumn{5}{|l|}{ Alcohol consumption ${ }^{d}$} \\
\hline Nondrinker during last 12 months & 18.18 & 18.17 & 22.78 & 24.41 \\
\hline Binge drinker & 15.98 & 17.57 & 23.64 & 28.02 \\
\hline Regular drinker & 11.40 & 11.02 & 19.07 & 19.84 \\
\hline Occasional drinker & 18.13 & 17.38 & 24.31 & 23.83 \\
\hline \multicolumn{5}{|l|}{ Smoking status ${ }^{c}$} \\
\hline Current smoker & 21.26 & 21.92 & 25.60 & 28.93 \\
\hline Former smoker & 12.99 & 12.65 & 20.52 & 21.95 \\
\hline Never smoker & 12.00 & 11.65 & 18.84 & 19.97 \\
\hline \multicolumn{5}{|l|}{ Physical activityb } \\
\hline Adequate activity & 9.70 & 8.58 & 16.82 & 18.50 \\
\hline Low activity & 14.95 & 14.86 & 21.90 & 23.23 \\
\hline \multicolumn{5}{|l|}{ Loneliness $^{\mathrm{e}}$} \\
\hline Yes & 23.68 & 24.23 & 32.35 & 33.94 \\
\hline No & 6.72 & 5.71 & 12.72 & 6.98 \\
\hline
\end{tabular}

${ }^{a}$ Number of participants in the longitudinal data with a depressive symptom score at all time points. ${ }^{b}$ Data for annual household income (Canadian dollars), social participation, number of chronic conditions and physical activity variables were not collected in the COVID-19 baseline and exit surveys; we used CLSA first follow-up values for the COVID-19 sample. 'Data for number of people living in the same household, dwelling type, living area and smoking status were not collected in the COVID-19 exit questionnaire; we used COVID-19 baseline values for the COVID-19 exit sample. ${ }^{\mathrm{D} D a t a}$ for alcohol consumption were not collected in the COVID-19 baseline survey; we used CLSA first follow-up values for the COVID-19 sample. 'Data for loneliness were not collected in the CLSA baseline survey; we used CLSA follow-up 1 values for the CLSA baseline and COVID-19 baseline sample. Data are shown as the percentage of participants. 
have reported worse mental health outcomes during the early weeks of the pandemic, some sources suggest a gradual decrease in anxiety and depressive symptoms during lockdown ${ }^{2}$. Some evidence also suggests that older individuals did not experience poorer psychological well-being than comparatively younger individuals ${ }^{11}$. However, social participation and loneliness were identified as important risk factors for psychological well-being, and it is these factors that have been impacted during the pandemic, especially for older adults. Therefore, it remains to be explained whether mental health continued to deteriorate during the initial lockdown (March-December 2020) or whether there were signs of stabilization or improvement in the mental health of community-dwelling middle-aged and older adults. Further, it remains to be clarified whether the risk factors had a differential effect on the psychological well-being of older adults compared with middle-aged adults or whether the impact was consistent across the adult lifespan. The purpose of this study was to examine the relationship of social determinants and healthrelated factors with changes in the prevalence of depressive symptoms during the initial lockdown and after reopening following the first wave in Canada and to evaluate the impact of loneliness and pandemic-related stressors on the severity and trajectory of depressive symptoms in middle-aged and older adults.

\section{Results}

Descriptive statistics. Cross-sectional descriptive characteristics of participants in the Canadian Longitudinal Study on Aging (CLSA) baseline $(n=51,338)$, first follow-up $(n=44,817)$, and COVID-19 baseline $(n=28,559)$ and exit $(n=24,114)$ surveys are provided in Supplementary Table 1 . Of the total participants recruited at CLSA baseline, $50.95 \%$ were female, $41.87 \%$ were aged 65 years and older, and $68.52 \%$ had an annual household income of CDN $\$ 50,000$ or more. Of the 28,559 participants recruited into the COVID-19 baseline survey, $0.34 \%$ were confirmed cases, $0.23 \%$ were probable cases and $9.34 \%$ were suspected cases of COVID-19. At the COVID-19 baseline survey, $21.15 \%$ of participants reported health-related concerns, $51.58 \%$ had difficulties in accessing resources, $7.17 \%$ experienced verbal or physical conflict, $20.91 \%$ reported caregiving challenges and $71.40 \%$ reported separation from family. The prevalence of depressive symptoms increased from $16.38 \%$ at CLSA baseline to $21.14 \%$ during the initial lockdown of the pandemic and was $21.96 \%$ at the time of administering the COVID-19 exit survey when there was a gradual lifting of the public health restrictions. The distributions of the prevalence of depressive symptoms by social determinants and health-related factors before and during the pandemic are presented in Table 1.

Sociodemographic factors and change in depressive symptoms. Results from weighted generalized estimating equation (WGEE) models examining the change in prevalence of depressive symptoms over time after adjusting for covariates are presented in Table 2. Overall, participants had approximately two times higher odds of depressive symptoms during the initial wave of the COVID19 pandemic compared with the pre-COVID-19 period. There was also evidence of differential effects by social determinants and health-related factors when examining effect modification with time period, demonstrating that some subgroups were more vulnerable to poor mental health. Our results showed an inverse association between annual income and depressive symptoms over time, where the lower the income, the greater the increase in the odds of depressive symptoms during the pandemic when compared with the highest income group before the pandemic. The odds of depressive symptoms during the initial lockdown increased by 3.35 times ( $95 \%$ confidence interval (CI): $2.81,3.99$ ) for individuals who had an annual household income of less than CDN $\$ 20,000$ and by 3.03 times $(95 \% \mathrm{CI}: 2.71,3.38)$ for those with income of CDN $\$ 20,000$ or greater and less than CDN $\$ 50,000$, relative to those with an income of $\mathrm{CDN} \$ 150,000$ or more in the pre-COVID-19 period.

Health-related risk factors including loneliness, pre-existing chronic conditions and low social participation were significant determinants of increase in the prevalence of depressive symptoms during the pandemic. The odds of depressive symptoms during the initial lockdown increased 6.75 times (95\% CI: 6.34, 7.18) for individuals who reported feelings of loneliness compared with those who did not report loneliness before the pandemic and 2.36 times (95\% CI: 2.18, 2.56) for individuals with lower social participation before the pandemic compared with those with higher social participation before the pandemic. Further, the odds of depressive symptoms during the initial lockdown increased for individuals living with a greater number of chronic conditions (three or more chronic conditions: odds ratio (OR): $3.92,95 \%$ CI: 3.58, 4.30; two chronic conditions: OR: 3.18 , 95\% CI: 2.89 , 3.51; one chronic condition: OR: $2.56,95 \% \mathrm{CI}: 2.34,2.81)$ compared with those with no chronic conditions before the pandemic. Overall, the odds of depressive symptoms remained elevated for all subgroups at the COVID-19 exit survey when compared with reference groups before the pandemic. Our results also showed evidence of differential effects by sex, living arrangements and health behaviors when interactions were examined with time period (Supplementary Table 2). We explored interactions of age with risk factors, including number of chronic conditions and loneliness, and found the results to be consistent with the trends reported for the main effects and time period by risk factor interactions (Supplementary Table 3). As part of a sensitivity analysis, we also examined the association stratified by age groups and found the results to be in agreement with those reported for the entire sample (Supplementary Table 4).

COVID-19 stressors and depressive symptom trajectories. The group-based trajectory model identified three distinct trajectory groups: one with a linear term and the other two with a quadratic term. The three depressive symptom trajectories were labeled as 'high-increasing', 'moderate-increasing' and 'low-consistent'. As depicted in Fig. 1, 8.0\% of participants showed high-increasing, $35.5 \%$ showed moderate-increasing and $56.6 \%$ showed low-consistent patterns of depressive symptoms over time. The linear change estimate was statistically significant for the low-consistent trajectory, while both linear and quadratic changes were statistically significant for the high-increasing and moderate-increasing trajectories.

Results from multivariable analysis evaluating the impact of loneliness and COVID-19 stressors on the severity and trajectory of depressive symptoms are presented in Table 3. The odds of a highincreasing trajectory were greater than for a low-consistent trajectory for adults who had experienced verbal or physical conflict (OR: 8.34; 95\% CI: 6.65, 10.45), reported loss of income or were unable to access resources (OR: 2.81; 95\% CI: 2.43, 3.25), had health-related concerns (OR: $2.47 ; 95 \%$ CI: 2.12, 2.88), were unable to provide care or spent more time in caregiving responsibilities (OR: 1.86; 95\% CI: $1.58,2.18$ ) and were separated from family (OR: $1.49 ; 95 \%$ CI: 1.28 , 1.73) during the pandemic. Further, loneliness was associated with membership in the high-increasing and moderate-increasing trajectory groups. Participants who reported being lonely were 15.50 times (95\% CI: 13.17, 18.25) more likely to be in the high-increasing trajectory group and 5.41 times (95\% CI: 4.94, 5.93) more likely to be in the moderate-increasing trajectory group compared with the low-consistent group. Social determinants and health-related factors were also significantly associated with the severity of depressive symptom trajectories (Table 3). Using latent class growth modeling (LCGM), we also explored the impact of the COVID-19 pandemic stressors and loneliness on depression subcomponents, including depressed affect, somatic symptoms and positive affect, and found the results to be largely consistent with those observed for 
Table 2 | Association of sociodemographic and health-related factors with depressive symptoms during the COVID-19 pandemic (weighted GEE models) $(n=37,111)^{\text {a }}$

\begin{tabular}{|c|c|c|c|c|c|}
\hline Factor & $\begin{array}{l}\text { Main } \\
\text { effects }\end{array}$ & $\begin{array}{l}\text { Annual household } \\
\text { income by period } \\
\text { interaction }\end{array}$ & $\begin{array}{l}\text { Social participation by } \\
\text { period interaction }\end{array}$ & $\begin{array}{l}\text { Chronic conditions by } \\
\text { period interaction }\end{array}$ & $\begin{array}{l}\text { Loneliness by } \\
\text { period interaction }\end{array}$ \\
\hline \multicolumn{6}{|l|}{ Period } \\
\hline $\begin{array}{l}\text { COVID-19 baseline versus } \\
\text { pre-COVID-19 }\end{array}$ & $\begin{array}{l}1.84(1.77 \\
1.91)\end{array}$ & $3.03(2.74,3.35)$ & $2.00(1.91,2.09)$ & $2.18(2.00,2.39)$ & $2.31(2.18,2.45)$ \\
\hline COVID-19 exit versus pre-COVID-19 & $\begin{array}{l}1.99(1.91 \\
2.07)\end{array}$ & $3.17(2.85,3.52)$ & $2.15(2.05,2.25)$ & $2.36(2.15,2.59)$ & $2.57(2.42,2.73)$ \\
\hline \multicolumn{6}{|l|}{ Age group ${ }^{b}$} \\
\hline$<55$ years versus $\geq 75$ years & $\begin{array}{l}1.79(1.65 \\
1.94)\end{array}$ & $1.86(1.71,2.02)$ & $1.79(1.65,1.94)$ & $1.83(1.69,1.99)$ & $1.79(1.65,1.95)$ \\
\hline $55-64$ years versus $\geq 75$ years & $\begin{array}{l}1.55(1.45 \\
1.65)\end{array}$ & $1.50(1.41,1.60)$ & $1.55(1.45,1.66)$ & $1.52(1.43,1.63)$ & $1.55(1.45,1.65)$ \\
\hline $65-74$ years versus $\geq 75$ years & $\begin{array}{l}1.19(1.12 \\
1.26)\end{array}$ & $1.15(1.08,1.22)$ & $1.19(1.12,1.26)$ & $1.17(1.10,1.24)$ & $1.19(1.12,1.26)$ \\
\hline \multicolumn{6}{|l|}{ Sex } \\
\hline Female versus male & $\begin{array}{l}1.49(1.42 \\
1.56)\end{array}$ & $1.49(1.42,1.57)$ & $1.49(1.42,1.57)$ & $1.49(1.42,1.57)$ & $1.49(1.42,1.56)$ \\
\hline \multicolumn{6}{|l|}{ Ethnicity } \\
\hline Nonwhite versus white & $\begin{array}{l}1.05(0.96 \\
1.15)\end{array}$ & $1.05(0.96,1.15)$ & $1.05(0.96,1.15)$ & $1.05(0.96,1.15)$ & $1.05(0.96,1.15)$ \\
\hline \multicolumn{6}{|l|}{ Annual household income } \\
\hline$<\$ 20,000$ versus $\geq \$ 150,000$ & $\begin{array}{l}1.56(1.38 \\
1.76)\end{array}$ & $2.97(2.62,3.37)$ & $1.56(1.38,1.76)$ & $1.56(1.38,1.76)$ & $1.55(1.37,1.75)$ \\
\hline $\begin{array}{l}\geq \$ 20,000 \text { to }<\$ 50,000 \text { versus } \\
\geq \$ 150,000\end{array}$ & $\begin{array}{l}1.25(1.15 \\
1.36)\end{array}$ & $1.95(1.77,2.14)$ & $1.25(1.15,1.36)$ & $1.25(1.15,1.36)$ & $1.25(1.14,1.36)$ \\
\hline $\begin{array}{l}\geq \$ 50,000 \text { to }<\$ 100,000 \text { versus } \\
\geq \$ 150,000\end{array}$ & $\begin{array}{l}1.08(1.01 \\
1.17)\end{array}$ & $1.46(1.34,1.60)$ & $1.08(1.01,1.17)$ & $1.08(1.01,1.17)$ & $1.08(1.10,1.16)$ \\
\hline $\begin{array}{l}\geq \$ 100,000 \text { to }<\$ 150,000 \text { versus } \\
\geq \$ 150,000\end{array}$ & $\begin{array}{l}1.02(0.94 \\
1.10)\end{array}$ & $1.27(1.16,1.40)$ & $1.02(0.94,1.10)$ & $1.02(0.94,1.10)$ & $1.01(0.94,1.10)$ \\
\hline \multicolumn{6}{|l|}{ Social participation } \\
\hline Low versus high social participation & $\begin{array}{l}1.36(1.29 \\
1.44)\end{array}$ & $1.36(1.28,1.44)$ & $1.63(1.551 .73)$ & $1.36(1.28,1.44)$ & $1.35(1.28,1.43)$ \\
\hline \multicolumn{6}{|l|}{$\begin{array}{l}\text { Number of people living in the same } \\
\text { household }\end{array}$} \\
\hline Living alone versus not living alone & $\begin{array}{l}0.94(0.89 \\
1.01)\end{array}$ & $0.94(0.89,1.01)$ & $0.94(0.89,1.01)$ & $0.94(0.89,1.00)$ & $0.94(0.89,1.01)$ \\
\hline \multicolumn{6}{|l|}{ Dwelling type } \\
\hline $\begin{array}{l}\text { Apartment or condominium versus } \\
\text { house }\end{array}$ & $\begin{array}{l}1.16(1.09 \\
1.24)\end{array}$ & $1.16(1.08,1.23)$ & $1.16(1.09,1.24)$ & $1.16(1.09,1.24)$ & $1.17(1.09,1.24)$ \\
\hline Other versus house & $\begin{array}{l}1.34(1.07 \\
1.67)\end{array}$ & $1.34(1.08,1.66)$ & $1.34(1.08,1.67)$ & $1.34(1.07,1.66)$ & $1.34(1.07,1.67)$ \\
\hline \multicolumn{6}{|l|}{ Living area } \\
\hline Urban versus rural & $\begin{array}{l}1.07(1.01 \\
1.14)\end{array}$ & $1.07(1.01,1.14)$ & $1.07(1.01,1.14)$ & $1.07(1.01,1.14)$ & $1.07(1.01,1.14)$ \\
\hline \multicolumn{6}{|l|}{ Number of chronic conditions } \\
\hline One versus none & $\begin{array}{l}1.22(1.14 \\
1.31)\end{array}$ & $1.23(1.14,1.31)$ & $1.22(1.14,1.31)$ & $1.29(1.19,1.39)$ & $1.22(1.14,1.31)$ \\
\hline Two versus none & $\begin{array}{l}1.50(1.40 \\
1.62)\end{array}$ & $1.51(1.41,1.63)$ & $1.50(1.40,1.62)$ & $1.72(1.59,1.86)$ & $1.50(1.39,1.61)$ \\
\hline Three or more versus none & $\begin{array}{l}2.01(1.88 \\
2.16)\end{array}$ & $2.02(1.88,2.17)$ & $2.01(1.87,2.16)$ & $2.42(2.23,2.61)$ & $2.01(1.87,2.16)$ \\
\hline Alcohol consumption & & & & & \\
\hline
\end{tabular}


Table 2 | Association of sociodemographic and health-related factors with depressive symptoms during the COVID-19 pandemic (weighted GEE models) $(n=37,111)^{\text {a }}$ (continued)

\begin{tabular}{|c|c|c|c|c|c|}
\hline Factor & $\begin{array}{l}\text { Main } \\
\text { effects }\end{array}$ & $\begin{array}{l}\text { Annual household } \\
\text { income by period } \\
\text { interaction }\end{array}$ & $\begin{array}{l}\text { Social participation by } \\
\text { period interaction }\end{array}$ & $\begin{array}{l}\text { Chronic conditions by } \\
\text { period interaction }\end{array}$ & $\begin{array}{l}\text { Loneliness by } \\
\text { period interaction }\end{array}$ \\
\hline Binge drinker versus nondrinker & $\begin{array}{l}1.06(0.94 \\
1.19)\end{array}$ & $1.06(0.94,1.19)_{-}$ & $1.06(0.95,1.20)$ & $1.06(0.94,1.19)$ & $1.07(0.95,1.20)$ \\
\hline Regular drinker versus nondrinker & $\begin{array}{l}0.85(0.79 \\
0.91)\end{array}$ & $0.85(0.79,0.91)$ & $0.85(0.79,0.91)$ & $0.85(0.79,0.91)$ & $0.85(0.80,0.91)$ \\
\hline Occasional drinker versus nondrinker & $\begin{array}{l}0.86(0.79 \\
0.93)\end{array}$ & $0.86(0.79,0.94)$ & $0.86(0.79,0.93)$ & $0.86(0.79,0.93)$ & $0.86(0.79,0.94)$ \\
\hline \multicolumn{6}{|l|}{ Smoking status } \\
\hline Current smoker versus never smoker & $\begin{array}{l}1.47(1.35 \\
1.61)\end{array}$ & $1.47(1.35,1.61)$ & $1.47(1.35,1.60)$ & $1.47(1.35,1.61)$ & $1.47(1.35,1.60)$ \\
\hline Former smoker versus never smoker & $\begin{array}{l}1.14(1.09 \\
1.21)\end{array}$ & $1.15(1.09,1.21)$ & $1.14(1.09,1.21)$ & $1.15(1.09,1.21)$ & $1.14(1.09,1.21)$ \\
\hline \multicolumn{6}{|l|}{ Physical activity } \\
\hline Low activity versus adequate activity & $\begin{array}{l}1.15(1.09 \\
1.21)\end{array}$ & $1.15(1.09,1.21)$ & $1.15(1.09,1.21)$ & $1.15(1.09,1.21)$ & $1.15(1.09,1.21)$ \\
\hline \multicolumn{6}{|l|}{ Loneliness } \\
\hline Yes versus no & $\begin{array}{l}3.25(3.10, \\
3.41)\end{array}$ & $3.25(3.10,3.41)$ & $3.25(3.10,3.40)$ & $3.24(3.10,3.40)$ & $4.21(3.99,4.43)$ \\
\hline
\end{tabular}

Annual household income $\times$ period (period reference $=$ pre-COVID-19/annual household income reference $=\geq \$ 150,000$ )

$\begin{array}{ll}<\$ 20,000 \text { versus } \geq \$ 150,000 & 3.35(2.81,3.99) \\ \text { at COVID-19 baseline versus } & \\ \text { pre-COVID-19 } & 3.03(2.71,3.38) \\ \geq \$ 20,000 \text { to }<\$ 50,000 \text { versus } & \\ \geq \$ 150,000 \text { at COVID-19 baseline } & \\ \text { versus pre-COVID-19 } & \\ \geq \$ 50,000 \text { to }<\$ 100,000 \text { versus } & \\ \geq \$ 150,000 \text { at COVID-19 baseline } & \\ \text { versus pre-COVID-19 } & \\ \geq \$ 100,000 \text { to }<\$ 150,000 \text { versus } & \\ \geq \$ 150,000 \text { at COVID-19 baseline } & \\ \text { versus pre-COVID-19 } & \\ <\$ 20,000 \text { versus } \geq \$ 150,000 \text { at } & \\ C O V I D-19 \text { exit versus pre-COVID-19 } & 3.08(2.56,3.72) \\ \geq \$ 20,000 \text { to }<\$ 50,000 \text { versus } & \\ \geq \$ 150,000 \text { at COVID-19 exit versus } & 3.16(2.82,3.54) \\ \text { pre-COVID-19 } & \\ \geq \$ 50,000 \text { to }<\$ 100,000 \text { versus } & \\ \geq \$ 150,000 \text { at COVID-19 exit versus } & \\ \text { pre-COVID-19 } & \\ \geq \$ 100,000 \text { to }<\$ 150,000 \text { versus } & \\ \geq \$ 150,000 \text { at COVID-19 exit versus } & \\ \text { pre-COVID-19 } & 3.01(2.69,3.37)\end{array}$

Social participation $\times$ period (period reference $=$ pre-COVID-19/social participation reference $=$ high social participation) Low social participation versus high $2.36(2.18,2.56)$ social participation at COVID-19 baseline versus pre-COVID-19

Low social participation versus high social participation at COVID-19 exit versus pre-COVID-19

Number of chronic conditions $\times$ period (period reference $=$ pre-COVID-19/number of chronic conditions reference $=0$ ) 1 versus 0 at COVID-19 baseline $2.56(2.34,2.81)$ versus pre-COVID-19 
Table 2 | Association of sociodemographic and health-related factors with depressive symptoms during the COVID-19 pandemic (weighted GEE models) $(n=37,111)^{\text {a }}$ (continued)

\begin{tabular}{|c|c|c|c|c|c|}
\hline Factor & $\begin{array}{l}\text { Main } \\
\text { effects }\end{array}$ & $\begin{array}{l}\text { Annual household } \\
\text { income by period } \\
\text { interaction }\end{array}$ & $\begin{array}{l}\text { Social participation by } \\
\text { period interaction }\end{array}$ & $\begin{array}{l}\text { Chronic conditions by } \\
\text { period interaction }\end{array}$ & $\begin{array}{l}\text { Loneliness by } \\
\text { period interaction }\end{array}$ \\
\hline $\begin{array}{l}3+\text { versus } 0 \text { at COVID-19 baseline } \\
\text { versus pre-COVID-19 }\end{array}$ & & & & $3.92(3.58,4.30)$ & \\
\hline $\begin{array}{l}1 \text { versus } 0 \text { at COVID-19 exit versus } \\
\text { pre-COVID-19 }\end{array}$ & & & & $2.85(2.59,3.14)$ & \\
\hline $\begin{array}{l}3+\text { versus } 0 \text { at COVID-19 exit versus } \\
\text { pre-COVID-19 }\end{array}$ & & & & $4.35(3.95,4.78)$ & \\
\hline \multicolumn{6}{|c|}{ Loneliness $\times$ period $($ period reference $=$ pre-COVID-19/loneliness reference $=$ not feeling lonely) } \\
\hline $\begin{array}{l}\text { Feeling lonely versus not feeling } \\
\text { lonely at COVID-19 baseline versus } \\
\text { pre-COVID-19 }\end{array}$ & & & & & $6.75(6.34,7.18)$ \\
\hline
\end{tabular}

${ }^{a}$ We performed WGEE modeling on 39,604 participants, which includes individuals with a monotonic pattern of missing data for the depressive symptom variable. ${ }^{\mathrm{b}} \mathrm{Age}$ group is included as a time-varying variable; loneliness data are from the CLSA first follow-up and all other covariates are from the CLSA baseline data. Data are shown as $\mathrm{OR}(95 \% \mathrm{CI})$.

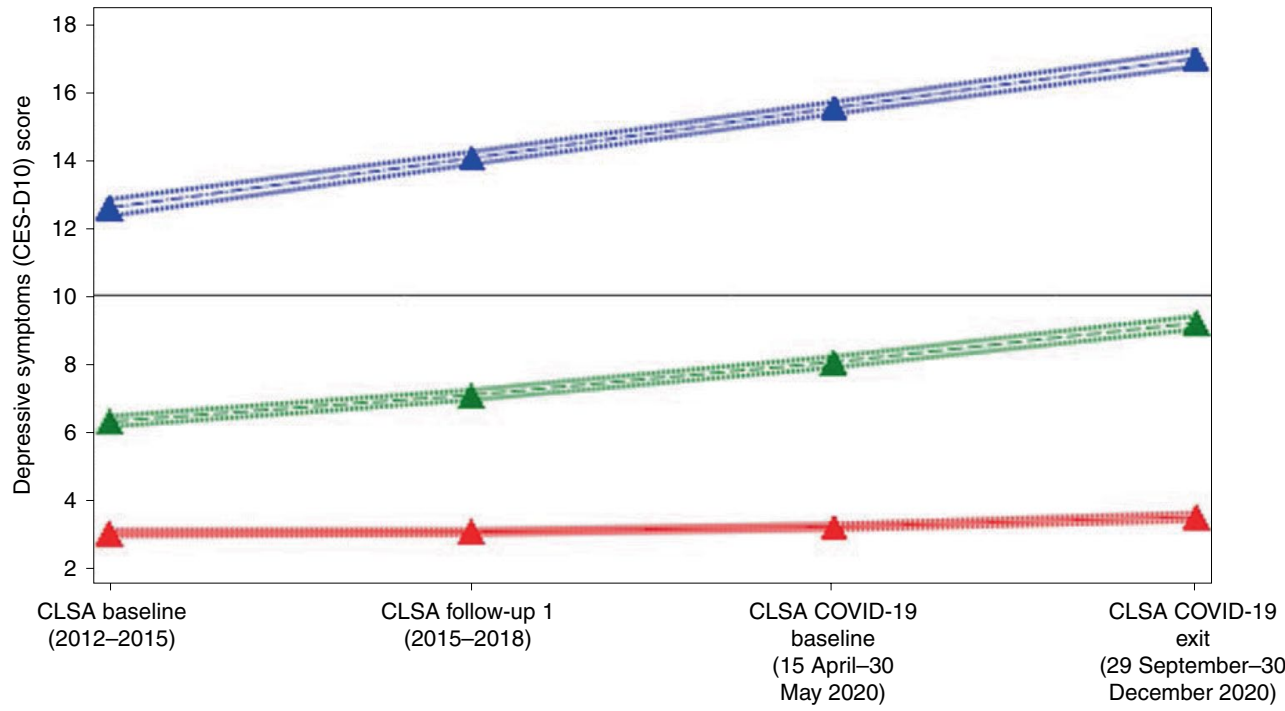

Fig. 1 | Average predicted depressive symptom score (dashed lines) with $95 \%$ Cls (dotted lines) during the COVID-19 pandemic $(n=20,478)$. The solid horizontal line indicates the threshold value (ten) for clinically significant depressive symptoms.

the overall depressive symptom score (Supplementary Table 5 and Supplementary Figs. 1-3).

\section{Discussion}

Using longitudinal data from a large, nationally generalizable sample of community-dwelling middle-aged and older adults, this study examined the association of social determinants and health-related factors with changes in the prevalence of depressive symptoms during the initial lockdown and after reopening following the first wave of COVID-19 in Canada and evaluated the impact of loneliness and pandemic-related stressors on the severity and trajectory of depressive symptoms. Our results showed that adults had twice the odds of depressive symptoms during the pandemic compared with the prepandemic period, with subgroups characterized by lower socioeconomic status and poorer health-related factors experiencing a greater impact across the age groups. More than four in ten adults showed a pattern of moderate or high levels of depressive symptoms at baseline that increased over time. Loneliness and COVID-19 stressors were significant predictors of worsening depressive symptom trajectories.

Social determinants were found to be the key drivers of mental health effects during the pandemic. In comparison with the low-consistent depressive symptom trajectory group, subgroups of adults with higher and increasing depressive symptoms were more 
Table 3 | Predictors of trajectory group membership associated with depressive symptom trajectory groups during the COVID-19 pandemic

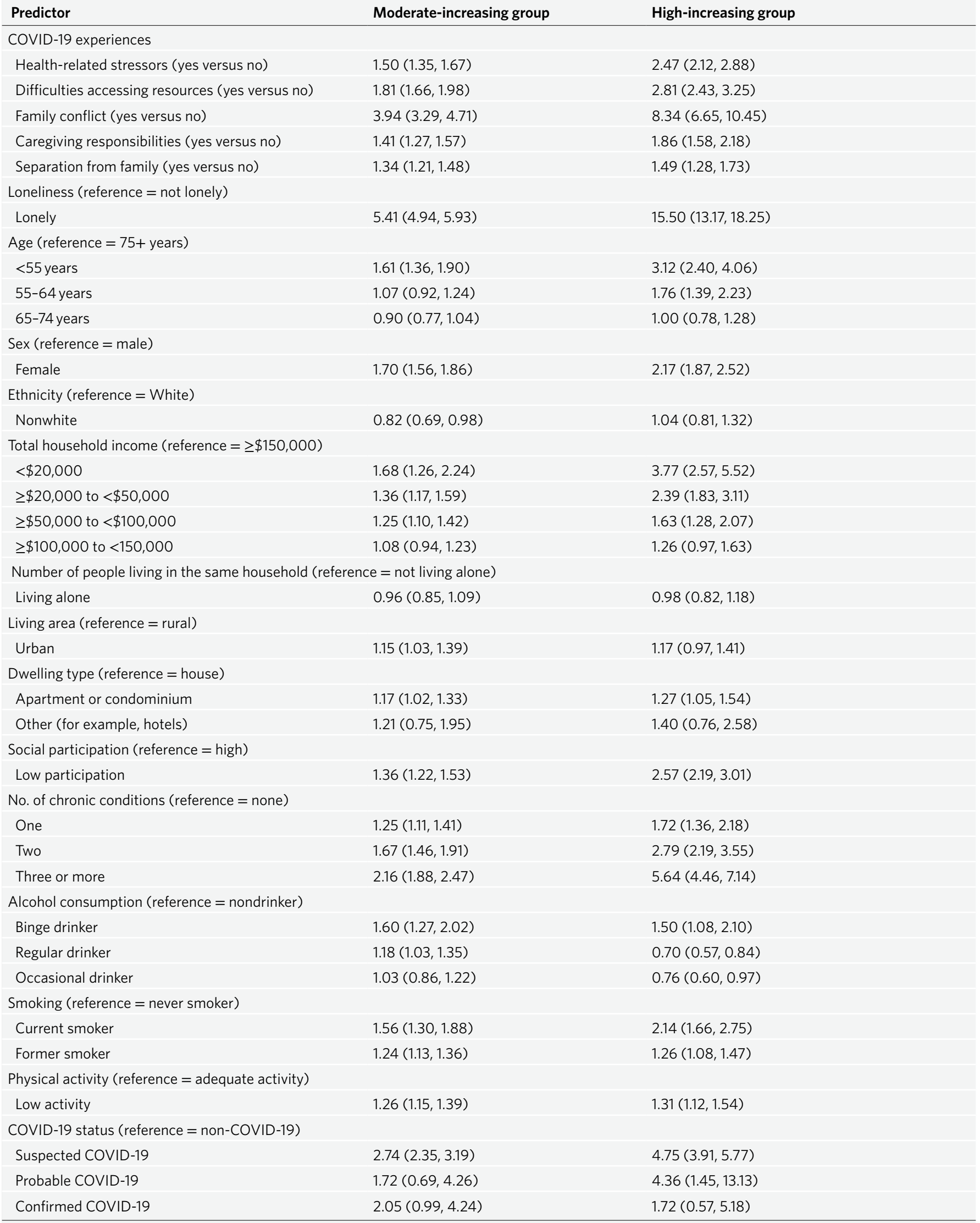

Data are shown as $\mathrm{OR}(95 \% \mathrm{Cl})$. 
likely to have lower income or report loss of income and difficulties in accessing resources including supplies or food, usual healthcare, prescription medications and treatments during the pandemic. These findings are consistent with previous research reporting unemployment, job insecurity, financial instability and food insecurity to be associated with stress, anxiety and depression ${ }^{12-15}$. Further, many individuals have been unable to access a range of healthcare services during the pandemic, including regular medical services and diagnostic procedures, elective surgical procedures and counseling services ${ }^{16,17}$. The inability to access healthcare services, especially for those living with pre-existing mental health conditions, frailty or multimorbidity, may lead to new or worsening depressive symptoms over time. In fact, our results showed that participants who reported health-related concerns during the pandemic and participants who had a greater number of pre-existing health conditions were more likely to have membership in the moderate or high and increasing depressive symptom trajectory groups compared with the low-consistent trajectory group.

Caregiving responsibilities, separation from family and loneliness were associated with a greater likelihood of membership in the moderate or high and increasing trajectory groups compared with the low-consistent group. Evidence indicates that caregivers have worse mental health and are more likely to report fatigue, sleep disturbance and anxiety than noncaregivers ${ }^{18,19}$. Caregiving responsibilities increased or intensified as informal caregivers may feel obligated to help care recipients with activities of daily living and manage health complications resulting from exposure to COVID-19 or due to disruptions in regular care access during the pandemic ${ }^{18}$. The pandemic made it physically, emotionally and economically more difficult for caregivers to provide care, thereby making them particularly more vulnerable to poor mental health ${ }^{19}$. Separation from family members due to the restrictions around social gatherings is also an important risk factor for poor mental health as it may limit access to informal caregivers and weaken social networks, resulting in increased social isolation and feelings of loneliness. There is evidence that loneliness and separation from family during the pandemic were associated with a higher risk of mood disorders and exacerbated pre-existing mental illness ${ }^{20,21}$. A greater proportion of females reported separation from family, increased time caregiving and inability to care for people who required assistance due to a health condition or limitation, which may partly explain the higher odds of depressive symptoms during the pandemic in females compared with males.

Adults who experienced verbal or physical conflict during the pandemic were more likely to show patterns of moderate or clinically high and increasing depressive symptoms. Studies have broadly demonstrated an increase in family conflict and interpersonal violence in the aftermath of natural disasters ${ }^{22,23}$. In the context of the COVID-19 pandemic, individual or simultaneous presence of risk factors such as unemployment, economic loss, lack of access to basic needs, lockdowns and social isolation, reduced healthcare service availability and the inability of individuals to temporarily escape abusive family members may increase the risk of, or exacerbate, emotional and physical family or partner conflict and may, in turn, increase the risk of worsening existing mental health problems ${ }^{24,25}$.

Together, our results suggest several targets for prevention and intervention. Responding to COVID-19 pandemic-related stressors and challenges requires a multifaceted approach that includes strategies that avoid disruption to healthcare access while following physical distancing guidelines (for example, telemedicine), enhance unemployment insurance, promote supportive workplace environments and policies, ensure adequate access to basic needs and resources, enable access to online and telephone support services and increase opportunities for safe social participation. Because disparities in mental health outcomes have persisted, interventions to mitigate the negative impact of the COVID-19 pandemic and lockdown on mental health should be targeted at those who are most at risk, including individuals with low socioeconomic status, those who are lonely and those living with multimorbidity. Our findings also indicate the need to identify and address concerns early to avoid worsening of depressive symptom trajectories.

The present study has several strengths, including the timing of data collection relative to the lockdown restrictions implemented in Canada and use of a large population-based sample to longitudinally examine social determinants, health-related factors and COVID-19 pandemic stressors associated with changes in the prevalence of depressive symptoms in middle-aged and older adults. However, it is important to note that our study sample did not include individuals residing in long-term care institutions, which may have led to underestimates of the prevalence of depressive symptoms and limits the generalizability of our findings to community-dwelling populations only. Further, a greater proportion of the participants who did not take part in the COVID-19 study were older, had lower annual income and had depressive symptoms (Supplementary Table 6), which may have impacted the validity of our findings. Differences in the method of administration of the ten-item Center for Epidemiologic Studies Short Depression Scale (CES-D10) questionnaire may also have impacted the results, as some participants completed the questionnaire in person whereas other participants completed the questionnaire over the telephone or online. However, the CES-D10 scale has been shown to perform well and had high internal consistency (range, $0.86-0.88$ ) in a sample of adults who completed the survey in person, over the telephone and online ${ }^{26}$. Measurement invariance of the CES-D10 has also been examined in the CLSA, and the results support the use of this scale in a heterogenous CLSA sample ${ }^{27}$. Also, information on social connectivity and network size during the pandemic was not available and therefore not examined in the analysis. We examined age as a time-varying covariate; however, other variables such as physical activity, social participation and alcohol consumption were not included as timevarying covariates in the analysis. Finally, the prevalence of depressive symptoms may have differed based on when, and during what phase, data collection was conducted during the pandemic.

Overall, the results of this study indicate that the COVID-19 pandemic has had a substantial negative impact on the mental health of middle-aged and older adults. Social determinants, loneliness and COVID-19 stressors were important predictors of increase in depressive symptoms during the pandemic. The disparities and patterns in the depressive symptom trajectories observed during the initial stages of the pandemic indicate that the negative mental health impacts of the pandemic persist and may worsen over the long term in the absence of effective interventions. Therefore, identifying and implementing intervention strategies, support and related public health measures for adults at higher risk of poor mental health trajectories may help alleviate the impact of the pandemic.

\section{Methods}

Data source and study participants. The study was approved by the Hamilton Integrated Research Ethics Board and by the research ethics boards of all the participating institutions across Canada. Written informed consent was obtained from the participants. Participants had the study and data collection procedures explained to them and had the opportunity to seek clarification before consenting. Participants provided separate consent for questionnaire and physical data collection, for biological sample collection and for linking their provincial health information to information collected by the CLSA. There was no direct compensation for the participants. The CLSA is a large, national, longitudinal cohort study that recruited 51,338 Canadian residents (25,183 males and 26,155 females) aged $45-85$ years at baseline (2012-2015) to be followed every 3 years, for at least 20 years or until death or loss to follow-up, to collect information on the changing biological, medical, psychological, social, lifestyle and economic aspects of people's lives. Participants were recruited from across the ten provinces through Statistics Canada's Canadian Community Health Survey, provincial healthcare registration databases and random digit dialing. Individuals residing in Canada's three territories, on First Nations reserves, in long-term care facilities, members of the armed forces, those who were unable to communicate in English or French 
and those with severe cognitive deficits were not eligible to participate in the study At each study visit, all participants provided data by questionnaires, but a subset of 30,097 participants living within 25-50 km of 1 of 11 Data Collection Sites (DCS) across seven Canadian provinces were also interviewed in their home and visited a DCS to provide a range of physical assessments and biological samples. The first follow-up was completed on 48,893 participants (95\% retention) in mid-2018, and the second follow-up is nearing completion (2018-2021). The CLSA design and methods have been described in detail elsewhere ${ }^{28}$

The CLSA participants were invited to participate in the CLSA COVID19 Questionnaire Study, which was launched on 15 April 2020 with the aim of understanding the epidemiology of COVID-19, including its impact on the mental health outcomes of middle-aged and older adults. Of the 51,388 CLSA participants, 8,638 were excluded because of death $(n=2,500)$, loss to follow-up $(n=3,406)$, requiring a proxy to participate $(n=318)$ or for other reasons such as unavailability of their contact information $(n=2,414)$. The remaining 42,700 participants were invited to participate by email to complete the web-based questionnaire $(n=34,498)$ or by telephone to complete the telephone interview with a trained CLSA interviewer $(n=8,202)$. During the recruitment process, another 189 participants were identified as deceased or requiring a proxy and therefore excluded. Of the 42,511 participants who were eligible to take part in the COVID-19 study, 28,559 (67.2\%) agreed to participate. Over the 9-month period, participants completed a 30-min questionnaire at baseline (15 April-30 May 2020), 10-min questionnaires weekly (four times by web) or biweekly (two times by phone), three monthly questionnaires and a final 30-min exit questionnaire (29 September-30 December 2020).

Measurement of study variables. Depressive symptoms. Screening for depressive symptoms in the CLSA was done using the CES-D10, which assesses depressive symptoms in the past week ${ }^{29}$. It includes three items on depressed affect, five items on somatic symptoms and two items on positive affect. Each item is scored from zero to three: rarely or never ( $<1$ day), some of the time ( $1-2$ days), occasionally (3-4 days) and all of the time (5-7 days). Scores for each participant were summed after reversing the positive affect items and can range between 0 and 30 with higher scores indicating a greater number of depressive symptoms. A positive screen for depressive symptoms was based on a score of ten or higher ${ }^{29}$. The CES-D10 has been shown to be reliable and valid in assessing depressive symptoms in adults, with internal consistency of 0.86 , test-retest reliability of 0.85 , convergent validity of 0.91 and divergence of 0.89 (refs. ${ }^{30,31}$ ).

COVID-19 experiences. The COVID-19 pandemic experiences and stressors were measured using a self-reported questionnaire administered at the COVID-19 baseline survey. Health-related stressors were identified by asking participants to indicate whether they were ill or if someone close to them was ill or had died due to COVID-19 or non-COVID-19-related reasons. Difficulties with accessing resources was identified by asking participants to indicate whether they had experienced loss of income, and difficulties in accessing necessary supplies, food and usual healthcare including prescription medications and treatments. Conflict was identified by asking participants to report whether they had experienced increased verbal or physical conflict. Separation from family was identified by asking participants to report whether they were separated from family during the pandemic. Caregiving experience assessed whether participants had spent increased time in caregiving or whether they were unable to care for people who required assistance due to a health condition or limitation. The pandemic experiences were grouped as 'yes' if the participant indicated at least one experience in the specific category or 'no' if the participant did not indicate any of the experiences in the specific category.

Covariates. The data from the CLSA baseline and first follow-up surveys were considered as being in the 'pre-COVID-19' period, and the time period variable for both these time points was coded as a zero and considered as the reference group in WGEE models. The time period was categorized as 'pre-COVID-19', 'COVID-19 baseline' and 'COVID-19 exit'. The WGEE model was adjusted for age $(<55,55-64$, 65-74 and $\geq 75$ years), sex, ethnicity (white or nonwhite), total annual household income $(\mathrm{CDN}<\$ 20,000, \$ 20,000$ to $<\$ 50,000, \$ 50,000$ to $<\$ 100,000, \$ 100,000$ to $<\$ 150,000$ and $\geq \$ 150,000$ ), dwelling type (house; apartment/condominium; or other type of dwelling including senior's housing, mobile home and hotel), living area (urban, rural), household composition (living alone, not living alone), loneliness (see definition below), alcohol consumption (never, occasional, binge or regular drinker), smoking status (never, former or current smoker), physical activity (see definition below), social participation (see definition below) and number of chronic conditions (see definition below). Loneliness was assessed using the Revised UCLA Loneliness Scale (R-UCLA) ${ }^{32}$. Participants were asked to indicate how often they felt left out, felt isolated from others and lacked companionship. Response options included 'hardly ever', 'some of the time' and 'often.' The total score may range from three to nine, and participants with a score of greater than three were classified as lonely $^{32}$. Physical activity was assessed using the Physical Activity Scale for the Elderly (PASE) and dichotomized as meeting the World Health Organization's age-specific guidelines for physical activity of at least $150 \mathrm{~min}$ of moderate-intensity or at least 75 min of vigorous-intensity physical activity per week ${ }^{33,34}$. Social participation was assessed by summing the frequency of involvement in eight categories to represent the number of community activities undertaken in a month. The social activities included family or friendship activities outside the household; church or religious activities; sports/physical activities with other people; educational or cultural activities involving other people; service club or fraternal organization activities; neighborhood, community or professional association activities; volunteer or charity work and other recreational activities involving other people ${ }^{35}$. The total score for social participation was categorized based on age- and sex-specific quintile into 'low' and 'high' social participation. The number of chronic conditions from ten disease categories including musculoskeletal, respiratory, cardiovascular, endocrine-metabolic, neurological, gastrointestinal, genitourinary, ophthalmologic, renal and cancer was summed up and categorized into 'none', 'one', 'two' and 'three or more' chronic conditions.

Statistical analysis. Descriptive statistics were reported at each time point. CLSA first follow-up data were used to impute a whole variable that was not assessed in the COVID-19 baseline and exit surveys, and COVID-19 baseline data were used to impute a variable that was not assessed in the COVID-19 exit survey. The WGEE was used to examine the change in prevalence of depressive symptoms over time. The WGEE can model longitudinal or clustered data and binary outcomes and can handle monotonic missing data appropriately when the data are missing at random ${ }^{36}$. We performed WGEE on 37,111 individuals, which includes those with a monotonic pattern of missing data for the depressive symptom variable. Interaction terms between time period and sociodemographic and health factors were assessed to determine how the change in depressive symptom prevalence over time depended on these factors. For each risk factor by time period interaction, ORs were reported for at-risk groups during the COVID-19 baseline and exit time points in comparison with the group that was least at risk before the pandemic. The WGEE models were adjusted for all the covariates listed above. Age was included as a time-varying covariate, loneliness was assessed at CLSA first followup and all other covariates were assessed at CLSA baseline.

LCGM, a semiparametric, group-based modeling strategy, was used to identify distinct classes of individuals who follow a similar pattern of depressive symptoms over the four time points and to examine the impact of loneliness and COVID19 experiences on the depressive symptoms trajectories ${ }^{37}$. The censored normal distribution was specified as the depressive symptoms score was modeled as a continuous variable to more accurately model data where floor and ceiling effects may be possible. Model selection involved testing different numbers and shapes of trajectory groups using statistical considerations and model parsimony. The best fitting model was identified by comparing the Akaike's information criterion (AIC) and Bayesian information criterion (BIC) values, with smaller values indicating a good fitting model. Quadratic and cubic terms that were not statistically significant were excluded from the model. Trajectories were modeled using the PROC TRAJ procedures in $\mathrm{SAS}^{37}$. Missing longitudinal data were handled in the PROC TRAJ procedure under the missing-at-random assumption, which permits patterns with missing data to borrow parameter information from patterns with more or complete data points through the latent variable ${ }^{38}$. In addition to the covariates listed above, the LCGM was adjusted for self-reported COVID-19 status based on the criteria adapted from the Public Health Agency of Canada and Centers for Disease Control and Prevention that were available during the initial wave of the pandemic ${ }^{39,40}$. Confirmed cases of COVID-19 included participants who reported testing positive for SARS-CoV-2 by nucleic acid amplification test. Probable cases of COVID-19 included (1) participants who had a laboratory test with fever (over $38^{\circ} \mathrm{C}$ ) or new onset or exacerbation of cough or who met the COVID-19 exposure criteria and were tested for COVID-19, but the results were inconclusive; or (2) participants who did not have a laboratory test but reported fever (over $38^{\circ} \mathrm{C}$ ) or a new onset or exacerbation of cough and had close contact with a confirmed COVID-19 case or lived or worked in a closed facility known to be experiencing an outbreak of COVID-19; (3) participants who were told by a healthcare provider that they had COVID-19 but did not have a confirmatory test. Suspected cases of COVID-19 included participants who reported two or more symptoms including fever, cough (dry or wet), runny nose, sore/scratchy throat, headache, chills or shivering, muscle and/or joint aches/pains, loss of smell or difficulty breathing and met the exposure criteria or had a close contact with a probable case of COVID19. OR and $95 \%$ CI values were reported, statistical significance was set at 0.05 for a two-tailed test and statistical analysis was conducted using SAS software v.9.4. CLSA has developed a core suite of software based on open-source code to collect data. The specific software used to collect data include ONYX (v.1.12.0), Limesurvey (v.3.7.1 with customizations) and PINE (v.2.7).

Reporting Summary. Further information on research design is available in the Nature Research Reporting Summary linked to this article.

\section{Data availability}

Data are available from the Canadian Longitudinal Study on Aging (www.clsa-elcv.ca) for researchers who meet the criteria for access to de-identified CLSA data.

\section{Code availability}

Custom code that supports the results of this study can be made available upon request from the corresponding author. 
Received: 24 March 2021; Accepted: 20 September 2021;

Published online: 25 November 2021

\section{References}

1. World Health Organization. WHO coronavirus (COVID-19) dashboard. https://covid19.who.int (2021)

2. Fancourt, D., Steptoe, A. \& Bu, F. Trajectories of anxiety and depressive symptoms during enforced isolation due to COVID-19 in England: a longitudinal observational study. Lancet Psychiatry 8, 141-149 (2021)

3. Government of Canada. Individual and community-based measures to mitigate the spread of COVID-19 in Canada. https://www.canada.ca/en/ public-health/services/diseases/2019-novel-coronavirus-infection/health professionals/public-health-measures-mitigate-covid-19.html (2021).

4. Mueller, A. L., McNamara, M. S. \& Sinclair, D. A. Why does COVID-19 disproportionately affect older people? Aging 12, 9959-9981 (2020).

5. Pierce, M. et al. Mental health before and during the COVID-19 pandemic: a longitudinal probability sample survey of the UK population. Lancet Psychiatry 7, 883-892 (2020).

6. Shanahan, L. et al. Emotional distress in young adults during the COVID-19 pandemic: evidence of risk and resilience from a longitudinal cohort study. Psychol. Med. 2020, 1-10 (2020).

7. Brooks, S. K. et al. The psychological impact of quarantine and how to reduce it: rapid review of the evidence. Lancet 395, 912-920 (2020).

8. Jalloh, M. F. et al. Impact of Ebola experiences and risk perceptions on mental health in Sierra Leone, July 2015. BMJ Glob. Health 3, e000471 (2018).

9. Hawryluck, L. et al. SARS control and psychological effects of quarantine, Toronto, Canada. Emerg. Infect. Dis. 10, 1206-1212 (2004).

10. Coyle, C. E. \& Dugan, E. Social isolation, loneliness and health among older adults. J. Aging Health 24, 1346-1363 (2012).

11. López, J. et al. Psychological well-being among older adults during the COVID-19 outbreak: a comparative study of the young-old and the old-old adults. Int. Psychogeriatr. 32, 1365-1370 (2020).

12. Wolfson, J. A., Garcia, T. \& Leung, C. W. Food insecurity is associated with depression, anxiety, and stress: evidence from the early days of the COVID-19 pandemic in the United States. Health Equity 5, 64-71 (2021)

13. Xiong, J. et al. Impact of COVID-19 pandemic on mental health in the general population: a systematic review. J. Affect. Disord. 277, 55-64 (2020).

14. Schmitz, N., Holley, P., Meng, X., Fish, L. \& Jedwab, J. COVID-19 and depressive symptoms: a community-based study in Quebec, Canada. Can. J. Psychiatry 65, 733-735 (2020).

15. Wilson, J. M. et al. Job insecurity and financial concern during the COVID-19 pandemic are associated with worse mental health. J. Occup. Environ. Med. 62, 686-691 (2020).

16. Yang, F., Dorrance, K. \& Aitken, N. The changes in health and well-being of Canadians with long-term conditions or disabilities since the start of the COVID-19 pandemic. https://www150.statcan.gc.ca/n1/pub/45-280001/2020001/article/00082-eng.htm (2020).

17. De Biase, S., Cook, L., Skelton, D. A., Witham, M. \& Ten Hove, R. The COVID-19 rehabilitation pandemic. Age Ageing 49, 696-700 (2020).

18. Park, S. S. Caregivers' mental health and somatic symptoms during COVID-19. Gerontol. B Psychol. Sci. Soc. Sci. 76, e235-e240 (2021).

19. Beach, S. R., Schulz, R., Donovan, H. \& Rosland, A. M. Family caregiving during the COVID-19 pandemic. Gerontologist 61, 650-660 (2021).

20. Holmes, E. A. et al. Multidisciplinary research priorities for the COVID-19 pandemic: a call for action for mental health science. Lancet Psychiatry 7 , 547-560 (2020).

21. Groarke, J. M. et al. Loneliness in the UK during the COVID-19 pandemic: cross-sectional results from the COVID-19 Psychological Wellbeing Study. PLoS ONE 15, e0239698 (2020).

22. Gearhart, S. et al. The impact of natural disasters on domestic violence: an analysis of reports of simple assault in Florida (1999-2007). Violence Gend. 5, 87-92 (2018).

23. Rezaeian, M. The association between natural disasters and violence: a systematic review of the literature and a call for more epidemiological studies. J. Res. Med. Sci. 18, 1103-1107 (2013).

24. Zhang, $\mathrm{H}$. The influence of the ongoing COVID-19 pandemic on family violence in China. J. Fam. Violence https://doi.org/10.1007/s10896-020-001968 (2020).

25. Gadermann, A. C. et al. Examining the impacts of the COVID-19 pandemic on family mental health in Canada: findings from a national cross-sectional study. BMJ Open 11, e042871 (2021).

26. Andresen, E. M., Byers, K., Friary, J., Kosloski, K. \& Montgomery, R. Performance of the 10-item Center for Epidemiologic Studies Depression Scale for caregiving research. SAGE Open Med. 1, 2050312113514576 (2013).

27. O'Connell, M. E. et al. P1-603: measurement invariance of the Centre for Epidemiological Studies Depression Scale 10-item short form (CES-D-10) in the Canadian Longitudinal Study on Aging. Alzheimers Dement. 14, P570 (2018).

28. Raina, P. et al. Cohort profile: the Canadian Longitudinal Study on Aging (CLSA). Int. J. Epidemiol. 48, 1752-1753 (2019).
29. Andresen, E. M., Malmgren, J. A., Carter, W. B. \& Patrick, D. L. Screening for depression in well older adults: evaluation of a short form of the CES-D (Center for Epidemiologic Studies Depression Scale). Am. J. Prev. Med. 10, 77-84 (1994).

30. Björgvinsson, T., Kertz, S. J., Bigda-Peyton, J. S., McCoy, K. L. \& Aderka, I. M. Psychometric properties of the CES-D-10 in a psychiatric sample. Assessment 20, 429-436 (2013).

31. Miller, W. C., Anton, H. A. \& Townson, A. F. Measurement properties of the CESD scale among individuals with spinal cord injury. Spinal Cord 46, 287-292 (2008)

32. Hughes, M. E., Waite, L. J., Hawkley, L. C. \& Cacioppo, J. T. A short scale for measuring loneliness in large surveys: results from two population-based studies. Res. Aging 26, 655-672 (2004).

33. Washburn, R. A., McAuley, E., Katula, J., Mihalko, S. L. \& Boileau, R. A. The Physical Activity Scale for the Elderly (PASE): evidence for validity. J. Clin. Epidemiol. 52, 643-651 (1999).

34. World Health Organization. Global recommendations on physical activity for health. https://www.who.int/publications/i/item/9789241599979 (2010).

35. Levasseur, M. et al. Associations between perceived proximity to neighborhood resources, disability, and social participation among community-dwelling older adults: results from the VoisiNuAge study. Arch. Phys. Med. Rehabil. 92, 1979-1986 (2011).

36. Salazar, A., Ojeda, B., Dueñas, M., Fernández, F. \& Failde, I. Simple generalized estimating equations (GEEs) and weighted generalized estimating equations (WGEEs) in longitudinal studies with dropouts: guidelines and implementation in R. Stat. Med. 35, 3424-3448 (2016).

37. Jones, B. L. \& Nagin, D. S. Advances in group-based trajectory modeling and an SAS procedure for estimating them. Sociol. Methods Res. 35, 542-571 (2007).

38. Dodge, H. H., Shen, C. \& Ganguli, M. Application of the pattern-mixture latent trajectory model in an epidemiological study with non-ignorable missingness. J. Data Sci. 6, 247-259 (2008).

39. Public Health Agency of Canada. Interim national case definition: coronavirus disease (COVID-19). https://open.canada.ca/data/en/dataset/722481c4-c44d4bb6-9ceb-002022d52f80 (Government of Canada, 2020).

40. Centers for Disease Control and Prevention. Coronavirus disease 2019 (COVID-19) 2020 interim case definition. https://wwwn.cdc.gov/nndss/ conditions/coronavirus-disease-2019-covid-19/case-definition/2020/08/05/ (2020)

\section{Acknowledgements}

Funding for support of the CLSA COVID-19 questionnaire-based study is provided by the Juravinski Research Institute, Faculty of Health Sciences, McMaster University, the Provost Fund from McMaster University, the McMaster Institute for Research on Aging, the Public Health Agency of Canada and the government of Nova Scotia. Funding for CLSA is provided by the government of Canada through the Canadian Institutes of Health Research (CIHR), under grant reference LSA 94473, and the Canada Foundation for Innovation and provincial governments (Newfoundland, Nova Scotia, Quebec, Ontario, Manitoba, Alberta and British Columbia). P.R. holds the Raymond and Margaret Labarge Chair in Optimal Aging and Knowledge Application for Optimal Aging, is the director of the McMaster Institute for Research on Aging and the Labarge Centre for Mobility in Aging and holds a Tier 1 Canada Research Chair in Geroscience. L.G. is supported by the McLaughlin Foundation Professorship in Population and Public Health. This research has been conducted using the CLSA Baseline Tracking Dataset version 3.7, Baseline Comprehensive Dataset version 5.1, Follow-up 1 Tracking Dataset version 2.2, Follow-up 1 Comprehensive Dataset version 3.0, CLSA Sample Weights version 1.2 and COVID-19 questionnaire data under application ID 21CON001.

\section{Author contributions}

P.R., C.W., L.G., S.K., J.M. and N.B. designed the CLSA COVID-19 study and contributed to the revision of the manuscript. U.E.O., N.S., D.J., G. Maimon and M.T. conducted the data analysis. P.R. and D.J. wrote the manuscript with input from all authors. The members of the CLSA team have contributed to the collection of the data across Canada.

\section{Competing interests}

The authors declare no competing interests.

\section{Additional information}

Supplementary information The online version contains supplementary material available at https://doi.org/10.1038/s43587-021-00128-1.

Correspondence and requests for materials should be addressed to Parminder Raina.

Peer review information Nature Aging thanks the anonymous reviewers for their contribution to the peer review of this work.

Reprints and permissions information is available at www.nature.com/reprints. Publisher's note Springer Nature remains neutral with regard to jurisdictional claims in published maps and institutional affiliations.

(c) The Author(s), under exclusive licence to Springer Nature America, Inc. 2021 


\section{CLSA team}

\section{Andrew Costa', Laura Anderson', Cynthia Balion'12, Asada Yukiko ${ }^{8}$, Benoît Cossette ${ }^{13}$, Melanie Levasseur ${ }^{13,14}$, Scott Hofer ${ }^{15}$, Theone Paterson ${ }^{15}$, David Hogan ${ }^{16}$, Teresa Liu-Ambrose ${ }^{17}$, Verena Menec ${ }^{18}$, Philip St. Johnn ${ }^{18,19}$, Gerald Mugford ${ }^{20}$, Zhiwei Gao ${ }^{20}$, Vanessa Taler ${ }^{21}$, Patrick Davidson ${ }^{21}$, Andrew Wister ${ }^{22}$ and Theodore $\operatorname{Cosco}^{22}$}

${ }^{12}$ Department of Pathology and Molecular Medicine, McMaster University, Hamilton, Ontario, Canada. ${ }^{13}$ Department of Community Health Sciences, University of Sherbrooke, Sherbrooke, Quebec, Canada. ${ }^{14}$ School of Readaptation, University of Sherbrooke, Québec, Quebec, Canada. ${ }^{15}$ Department of Psychology, University of Victoria, Victoria, British Columbia, Canada. ${ }^{16}$ Department of Medicine, University of Calgary, Calgary, Alberta, Canada. ${ }^{17}$ Physical Therapy, University of British Columbia, Vancouver, British Columbia, Canada. ${ }^{18}$ Department of Community Medicine, University of Manitoba, Winnipeg, Manitoba, Canada. ${ }^{19}$ Department of Geriatric Medicine, University of Manitoba, Winnipeg, Manitoba, Canada. ${ }^{20}$ Faculty of Medicine, Memorial University of Newfoundland, St. John's, Newfoundland and Labrador, Canada. ${ }^{21}$ School of Psychology, University of Ottawa, Ottawa, Canada. ${ }^{22}$ Department of Gerontology, and Gerontology Research Centre, Simon Fraser University, Vancouver, British Columbia, Canada. 


\section{Reporting Summary}

Nature Portfolio wishes to improve the reproducibility of the work that we publish. This form provides structure for consistency and transparency in reporting. For further information on Nature Portfolio policies, see our Editorial Policies and the Editorial Policy Checklist.

\section{Statistics}

For all statistical analyses, confirm that the following items are present in the figure legend, table legend, main text, or Methods section.

$\mathrm{n} / \mathrm{a} \mid$ Confirmed

$\bigotimes$ The exact sample size $(n)$ for each experimental group/condition, given as a discrete number and unit of measurement

$\square$ A statement on whether measurements were taken from distinct samples or whether the same sample was measured repeatedly

The statistical test(s) used AND whether they are one- or two-sided

Only common tests should be described solely by name; describe more complex techniques in the Methods section.

\ A description of all covariates tested

$\square$ A description of any assumptions or corrections, such as tests of normality and adjustment for multiple comparisons

A full description of the statistical parameters including central tendency (e.g. means) or other basic estimates (e.g. regression coefficient)

AND variation (e.g. standard deviation) or associated estimates of uncertainty (e.g. confidence intervals)

For null hypothesis testing, the test statistic (e.g. $F, t, r$ ) with confidence intervals, effect sizes, degrees of freedom and $P$ value noted

Give $P$ values as exact values whenever suitable.

Х $\square$ For Bayesian analysis, information on the choice of priors and Markov chain Monte Carlo settings

Х $\square$ For hierarchical and complex designs, identification of the appropriate level for tests and full reporting of outcomes

$\square \bigotimes$ Estimates of effect sizes (e.g. Cohen's $d$, Pearson's $r$ ), indicating how they were calculated

\section{Our web collection on statistics for biologists contains articles on many of the points above.}

\section{Software and code}

Policy information about availability of computer code

Data collection CLSA has developed core suite of software based on open source code to collect data. The specific software used to collect data include ONYX (version 1.12.0), Limesurvey (version 3.7.1 with customizations), and PINE (version 2.7). www.clsa-elcv.ca

Data analysis Statistical analysis was conducted using SAS software version 9.4. Custom code that supports the results of this study can be made available upon request from the corresponding author.

For manuscripts utilizing custom algorithms or software that are central to the research but not yet described in published literature, software must be made available to editors and

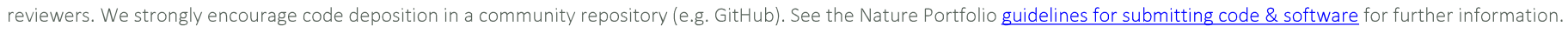

\section{Data}

Policy information about availability of data

All manuscripts must include a data availability statement. This statement should provide the following information, where applicable:

- Accession codes, unique identifiers, or web links for publicly available datasets

- A description of any restrictions on data availability

- For clinical datasets or third party data, please ensure that the statement adheres to our policy 
Please select the one below that is the best fit for your research. If you are not sure, read the appropriate sections before making your selection.

Life sciences

\section{Behavioural \& social sciences study design}

All studies must disclose on these points even when the disclosure is negative.

Study description

Research sample

Sampling strategy

Data collection

Timing

Data exclusions

Non-participation

Randomization
The current study is a quantitative study that is longitudinal in design. The Canadian Longitudinal Study on Aging (CLSA) recruited 51,388 community-dwelling adults from the 10 Canadian provinces at baseline (2011-2015) to be followed every 3 years, for at least 20 years or until death, to collect information on the changing biological, medical, psychological, social, lifestyle and economic aspects of people's lives. Of these participants, 28,559 were recruited to participate in the CLSA COVID-19 Study (April-December 2020). Longitudinal data from these studies were examined in the current study.

The Canadian Longitudinal Study on Aging (CLSA) is a large, national, longitudinal cohort study that recruited 51,338 Canadian residents aged 45-85 years at baseline (2011-2015). The middle-aged adults were included at baseline to capture mid-life experiences prospectively, since many important transitions that impact health outcomes later in life tend to occur during this period. Adults as old as 85 years were included to prospectively examine transitions into the final years of life. The first follow-up was completed on 48,893 participants (95\% retention) in mid-2018. A total of 42,511 participants from the core CLSA study were eligible to participate in the CLSA COVID-19 study of which 28,559 (67.2) agreed to participate. Of the total participants recruited at CLSA baseline, 50.95\% were females, $41.87 \%$ were aged 65 years and older, $92.68 \%$ were of European ethnic background, and $68.52 \%$ had total household income of $\$ 50,000$ or more.

A stratified, random sampling was used to sample participants into the core CLSA study. Two methods were used to recruit participants into the CLSA COVID-19 Study: via e-mail and by telephone. To calculate the CLSA sample size at baseline, the expected number of outcomes were estimated for each 3-year follow-up period based on age and sex specific incidence rates and after accounting for mortality and loss-to-follow-up. In the next step, the expected number of outcomes and a range of exposure/risk factor prevalence $(5 \%, 10 \%, 20 \%$, and $50 \%)$ were used to estimate the minimum detectable odds ratio between an exposure and outcome, after accounting for misclassification of the exposure and outcome. This iterative simulation-based approach demonstrated that the CLSA data is sufficiently powered to examine a wide range of associations, and especially those involving more common outcomes such as depressive symptoms.

The CLSA recruited 51,338 Canadian residents aged 45-85 years at baseline (2011-2015), to be followed every three years, for at least 20 years or until death or loss to follow-up, to collect information on the changing biological, medical, psychological, social, lifestyle and economic aspects of people's lives. At each study visit, all participants provide data by questionnaires but a subset of 30,097 participants living within 25-50 km of one of 11 Data Collection Sites (DCS) across seven Canadian provinces are also interviewed in their home and visit a DCS to provide a range of physical assessments and biological samples. The first follow-up was completed on 48,893 participants in mid-2018.

The CLSA COVID-19 questionnaire-based study was launched on April 15, 2020 to understand the epidemiology of COVID-19 including social and mental health consequences of the pandemic among older Canadians. Participants who had active email addresses were contacted by email and invited to participate via a web questionnaires $(n=34,498)$ and those without an active email address were invited to complete the interviews by telephone $(n=8,202)$ where information was entered for them by trained CLSA interviewers.

The CLSA baseline data were collected between 2011-2015. For participants who provided data through telephone interview, data collection took place between September 2011-December 2015, and for participants who provided data through in-home interview and at a data collection site, data collection took place between May 2012 and May 2015. The first follow-up was completed from 2015-2018 (Telephone interview from December 2015 to December 2018 and in-home interview and data collection site visit from July 2015 to July 2018).

The CLSA COVID-19 study was launched on April 15, 2020 and data were collected multiple time points over a 9-month period. The participants completed 10-minute questionnaires weekly ( 4 times by web) or biweekly ( 2 times by phone), 3 monthly questionnaires and a final 30-minute exit questionnaire between September and December of 2020.

No data were excluded from the analysis.

Of the 51,338 participants recruited at CLSA baseline, first follow-up was completed on 48,893 participants ( $95 \%$ retention) in mid-2018.

Of the 51,338 CLSA participants, 42,700 were invited to take part in the CLSA COVID-19 study. The 8,638 excluded individuals included those who had died $(n=2,500)$, withdrew prior to follow-up $2(n=3,406)$, required a proxy $(n=318)$, or for administrative reasons, the most common being that current contact information was not available $(n=2,414)$. During the recruitment process, an additional 166 participants were identified as deceased and 23 as needing a proxy to arrive at the overall eligible sample of 42,511, of which 28,559 (67.2\%) agreed to participate in the CLSA COVID-19 study.

n/a

Participants were not randomized into experimental groups. The Weighted Generalized Estimating Equations and Latent Class Growth Modeling techniques were used to answer the research questions after adjusting for the covariates. 


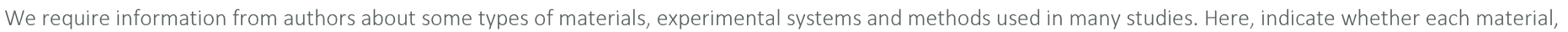

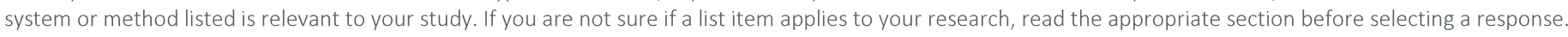

Materials \& experimental systems

$\mathrm{n} / \mathrm{a}$ Involved in the study

\ $\square$ Antibodies

\ $\square$ Eukaryotic cell lines

Х $\square$ Palaeontology and archaeology

\ $\square$ Animals and other organisms

$\square$ Wuman research participants

\ $\square$ Clinical data $\bigotimes \square$ Dual use research of concern

\begin{tabular}{l|l}
\multicolumn{2}{l}{ Methods } \\
\hline n/a & Involved in the study \\
$\square$ & $\square$ ChIP-seq \\
$\square$ & $\square$ Flow cytometry \\
$\square$ & $\square$ MRI-based neuroimaging
\end{tabular}

Human research participants

Policy information about studies involving human research participants

Population characteristics

See above.

Recruitment

The CLSA is a national cohort. A random sample of eligible households was contacted. If an eligible individual in the household was identified, they were asked to provide their contact information to the CLSA. Individuals who responded by providing their contact information were then contacted, and those who completed all required baseline interviews and assessments and provided written informed consent were enrolled into the CLSA cohort.

Our study sample did not include individuals residing in the Canada's three territories, on First Nations reserves, in long-term care facilities, members of the armed forces, those who were unable to communicate in English or French, and those with severe cognitive deficits, which may have underestimated the prevalence of depressive symptoms, and limit the generalizability of our findings to community-dwelling populations only.

Ethics oversight

The study was approved by the Hamilton Integrated Research Ethics Board and by research ethics boards of all the participating institutions across Canada. Informed consent was obtained from the participants. Participants were explained the study and data collection procedures and had the opportunity to seek clarification prior to consenting. Participants provided separate consents for questionnaire and physical data collection, biological sample collection, and for linking their provincial health information to information collected by the CLSA. 